\title{
Thermosphere and exosphere of hot Jupiters
}

\author{
Alain Lecavelier des Etangs \\ Institut d'astrophysique de Paris, CNRS/UPMC, 98bis bld Arago, F-75014 Paris, France
}

\begin{abstract}
Here we describe the observations and the resulting constraints on the upper atmosphere (thermosphere and exosphere) of hot Jupiters. In particular, observations and theoretical modeling of hot-Jupiter evaporation are described. The observations allowed the discovery that the planet orbiting HD209458 has an extended atmosphere of escaping hydrogen and showed the presence of oxygen and carbon at very high altitude. These observations give unique constraints on the escape rate and mechanism in the atmosphere of these planets. The most recent Lyman-alpha HST observations of HD189733b allow for the first time to compare the evaporation from two different planets in different environments. We present models to quantify the escape rate from the measured occultation depths, and an energy diagram to describe the evaporation state of hot Jupiters. Using this diagram, it is shown that few already known planets could be remnants of former giant planets.
\end{abstract}

\section{Thermosphere of HD $209458 \mathrm{~b}$ and HD $189733 \mathrm{~b}$}

The physical parameters of the upper atmospheres up to the exosphere (or the thermosphere) can be determined using absorption spectroscopy of transits. This technique has been developed in details for HD 209458 b (Sing et al. 2008a, 2008b; Lecavelier des Etangs et al. 2008b; Désert et al. 2008) where the temperature-pressure-altitude profile has been estimated from $\sim 0.1$ mbar to $\sim 50$ mbar. In particular, because the atmospheric scale height is directly related to the temperature, the temperature can be easily determined by measuring the variation of transit occultation depth as a function of wavelength. For instance, when detected, the slope of the Rayleigh scattering allows a direct determination of the temperature at the altitude where Rayleigh scattering is optically thick (Lecavelier des Etangs et al. 2008a, 2008b). For more details see the Sing et al. contribution in this volume.

\section{HD $209458 \mathrm{~b}$, an evaporating planet}

Transit observations have also revealed the evaporation of hot Jupiters close to their parent stars. For more than ten years, transit observations have allowed discoveries, detection, and characterization of extrasolar objects (Lecavelier des Etangs et al. 1995, 1997, 1999a, 1999b, 2005; Lamers et al. 1997; Nitschelm et al. 2000; Hébrard \& Lecavelier des Etangs 2006; Ehrenreich et al. 2006, 2007). In the recent discoveries, the evaporation of hot Jupiters opens a new field of research in the exoplanet field, starting with the observational discovery that HD20958b is evaporating (Vidal-Madjar et al. 2003, hereafter VM03). This discovery has been challenged by a recent work of BenJaffel (2007); but the apparent discrepancy has been solved and the result obtained from this observation data set is strengthened (Vidal-Madjar et al. 2008).

Three transits of HD209458b were surveyed with the STIS spectrograph on-board HST $\left(\sim 20 \mathrm{~km} \mathrm{~s}^{-1}\right.$ resolution). For each transit, three consecutive HST orbits were scheduled so that the first orbit ended before the first contact to serve as a reference, the two following orbits being partly or entirely within the transit. An average $15 \pm 4 \%(1 \sigma)$ 
relative intensity drop near the center of the Lyman- $\alpha$ line was observed during the transits. This is larger than expected for the atmosphere of a planet occulting only $\sim 1.5 \%$ of the star.

Because of the small distance $\left(8.5 \mathrm{R}_{*}\right)$ between the planet and the star (allowing an intense heating of the planet and its classification as a "hot Jupiter") the Roche lobe is only at 2.7 planetary radii (i.e. $3.6 \mathrm{R}_{\mathrm{Jup}}$ ). Filling up this lobe with hydrogen atoms gives a maximum absorption of $\sim 10 \%$ during planetary transits. Since a more important absorption was detected, hydrogen atoms cover a larger area corresponding to a spherical object of $4.3 \mathrm{R}_{\mathrm{Jup}}$. Observed beyond the Roche limit, these hydrogen atoms must be escaping the planet. Independently, the spectral absorption width, with blueshifted absorption up to $-130 \mathrm{~km} \mathrm{~s}^{-1}$, also shows that some hydrogen atoms have large velocities relative to the planet, exceeding the escape velocity. This further confirms that hydrogen atoms must be escaping the planetary atmosphere.

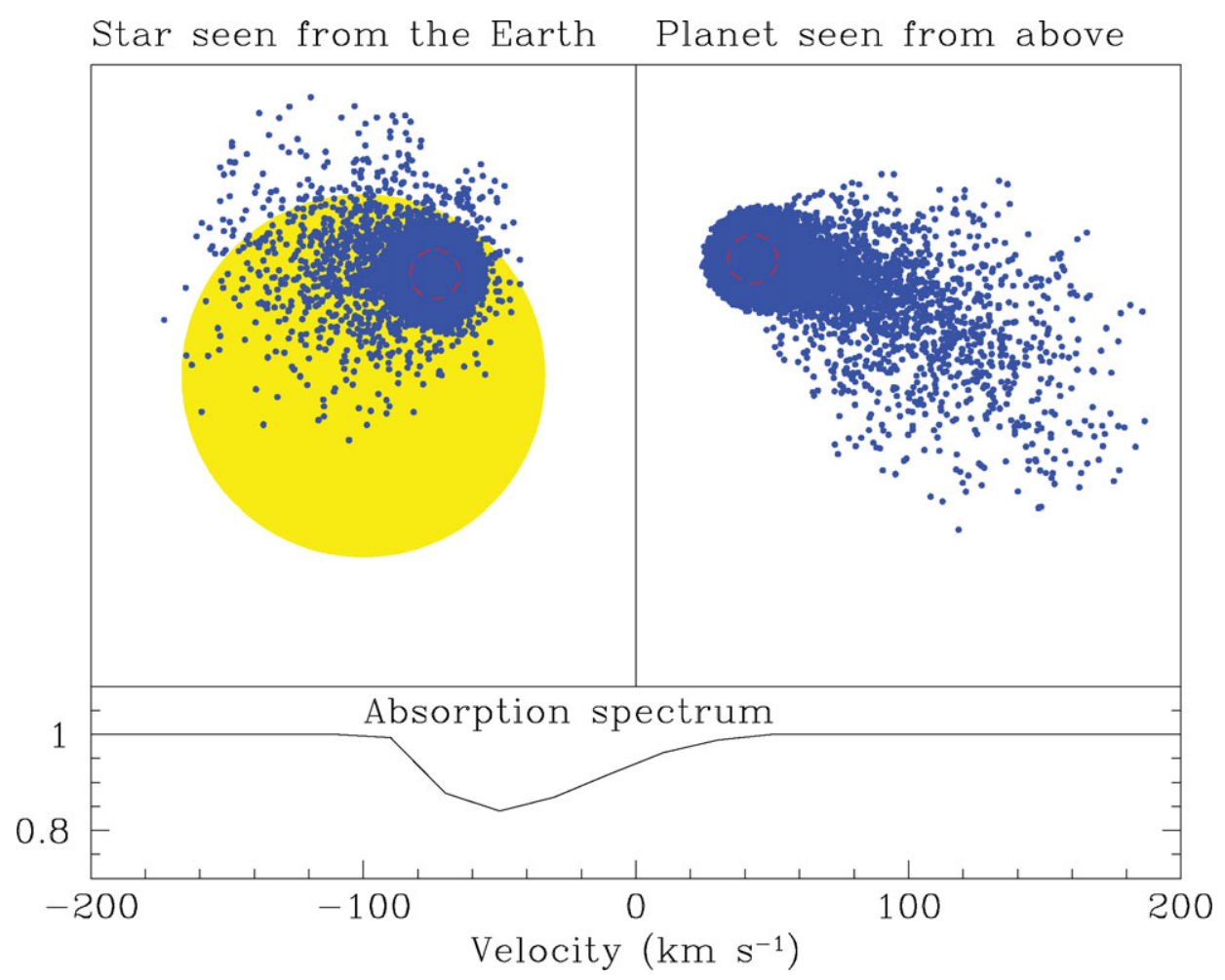

Figure 1. A numerical simulation of hydrogen atoms sensitive to radiation pressure (0.7 times the stellar gravitation) above an altitude of 0.5 times the Roche radius where the density is assumed to be $2 \times 10^{5} \mathrm{~cm}^{-3}$. It corresponds to an escape flux of $\sim 10^{10} \mathrm{~g} \mathrm{~s}^{-1}$. The mean ionization lifetime of escaping hydrogen atoms is 4 hours. The model yields an atom population in a curved cometary-like tail (see details in Vidal-Madjar \& Lecavelier 2004).

The observed $15 \%$ intensity drop could only be explained if hydrogen atoms are able to reach the Roche lobe of the planet and then escape. To evaluate the amount of escaping atoms, a particle simulation was built, in which hydrogen atoms are assumed to be sensitive to the stellar gravity and radiation pressure (see Fig. 2). In this simulation, escaping hydrogen atoms expand in an asymmetric cometary like tail and are progressively ionized when moving away from the planet. Atoms in the evaporating coma and 
tail cover a large area of the star. An escape flux of $\sim 10^{10} \mathrm{~g} \cdot \mathrm{s}^{-1}$ is needed to explain the observations. Taking into account the tidal forces and the temperature rise expected in the upper atmosphere, theoretical evaluations are in good agreement with the observed rate (see references in Sect 4 ).

\section{Hydrodynamical escape or "Blow-off"}

Four transits of HD 209458 b were then observed, again with the STIS spectrograph on board HST, but at lower resolution (Vidal-Madjar et al. 2004, hereafter VM04). The wavelength domain (1180-1710A) includes H I as well as C I , C II , C III , N v , O I , S I, Si III, S IV and Fe II lines. During the transits, absorptions are detected in H I , O I and C II $(5 \pm 2 \%, 10 \pm 3.5 \%$ and $6 \pm 3 \%$, respectively). No absorptions are detected for other lines. The $5 \%$ mean absorption over the whole H I Lyman-alpha line is consistent with the previous detection at higher resolution (VM03). The absorption depths in O I and $\mathrm{C}$ II show that oxygen and carbon are present in the extended upper atmosphere of HD 209458b. These species must be carried out up to the Roche lobe and beyond, most likely in a state of hydrodynamic escape.

\section{A diagram for the evaporation status of extrasolar planets}

The observational constraints given in previous sections have been used to develop a large number of models. These models aim at a better understanding of the observed escape rate and evaporation properties, and subsequently drawing the consequence on other planets and planetary systems (Lammer et al. 2003; Lecavelier des Etangs et al. 2004, 2007; Baraffe et al. 2004, 2005, 2006; Yelle 2004, 2006; Jaritz et al. 2004; Tian et al. 2005; Hubbard et al. 2005; Garcia-Munoz 2007). However, all the modeling efforts lead to the conclusion that most of the EUV and X-ray input energy by the harboring star is used by the atmosphere to escape the planet gravitational potential.

Therefore, to describe the evaporation status of the extrasolar planets, an energy diagram as been developed in which the potential energy of the planets is plotted versus the energy received by the upper atmosphere (Lecavelier des Etangs 2007). This description allows a quick estimate of both the escape rate of the atmospheric gas and the lifetime of a planet against the evaporation process. In the energy diagram, there is an evaporation-forbidden region in which a gaseous planet would evaporate in less than 5 billion years. With their observed characteristics, all extrasolar planets are found outside this evaporation-forbidden region (Fig. 2).

\section{Neptune-mass planets in the diagram}

A plot of the mass distribution of the extrasolar planets shows that Neptune-mass and Earth-mass planets play a particular role (Lecavelier des Etangs 2007). On June 25, with radial velocity searches, nineteen (19!) planets have been found with mass below $0.072 M_{\text {Jup }}$ (23 Earth-mass), while only one (1!) planet has been identified with mass in the range $0.072 M_{\text {jup }}-0.11 M_{\text {jup }}$ (23-35 Earth-mass). This gap is not a bias in the radial velocity searches, since more massive planets are easier to detect. This reveals the different nature of these Neptune-mass planets orbiting at short orbital distances. But their nature is still a matter of debate (Baraffe et al. 2005). In particular the question arises if they can be the remnants of evaporated more massive planets ("chthonian planets") as foreseen in Lecavelier des Etangs et al. (2004). Other possibilities include gaseous Neptune-like 


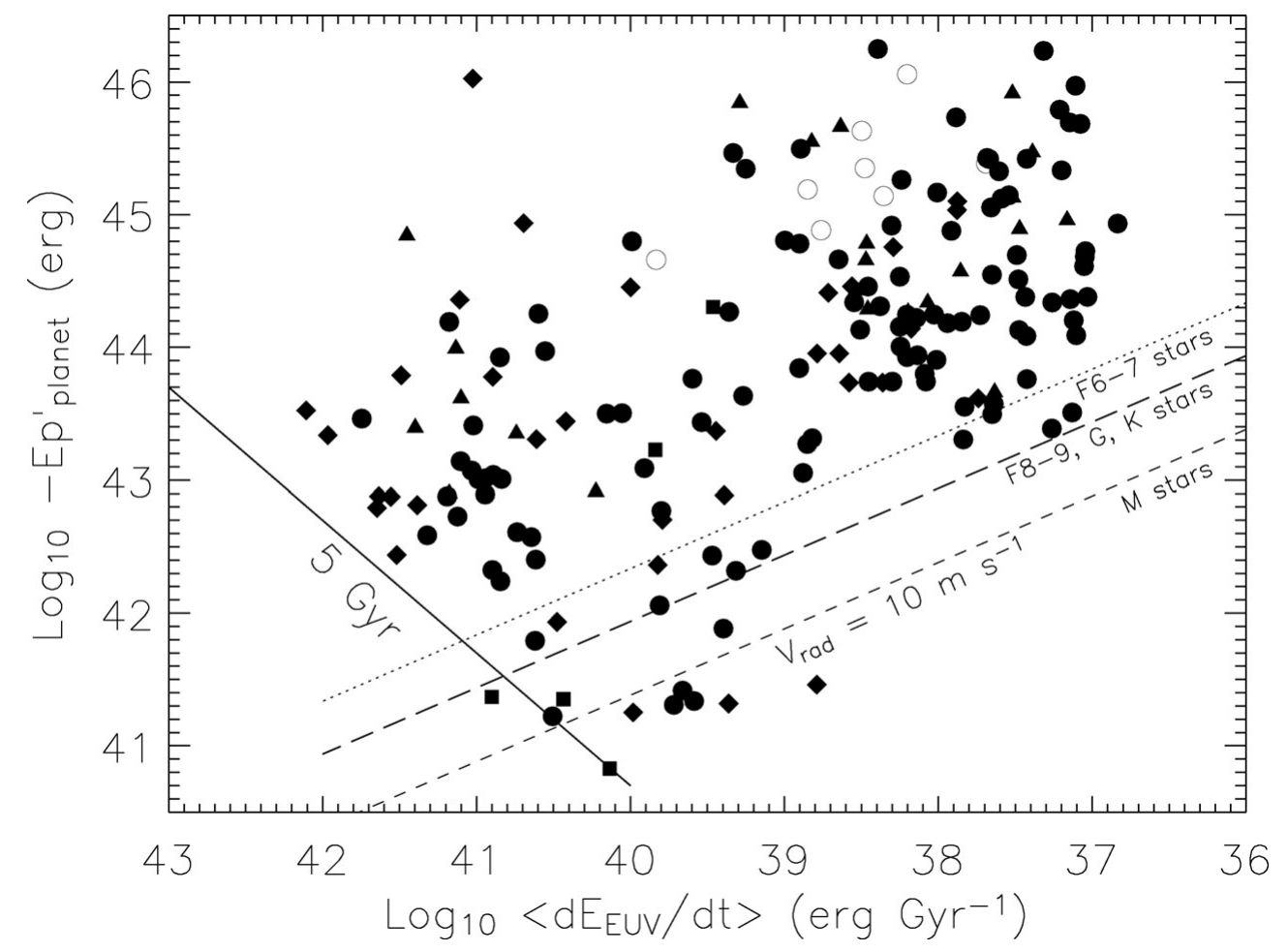

Figure 2. Plot of the potential energy of the extrasolar planets as a function of the mean EUV energy received per billion of years, $\left\langle d E_{\mathrm{EUV}} / d t\right\rangle$. To conserve the analogy with a period-mass diagram, the direction of the abscissa axis is chosen with the largest value of the mean energy flux toward the left. Identified planets are plotted with symbols depending on the type of the central star: triangles for $\mathrm{F}$ stars, filled circles for $\mathrm{G}$ stars, diamonds for $\mathrm{K}$ stars and squares for M stars; planets orbiting class III stars are plotted with empty circles. From the position in the diagram, the typical lifetime of a given planet can be rapidly extracted. If the mean energy flux $<d E_{\mathrm{EUV}} / d t>$ is given in unit of erg per billion years, and the potential energy is given in unit of erg, the simple ratio of both quantities provides the corresponding lifetime in billion of years. In the diagram, lifetime isochrones are straight lines. The lifetime of $5 \mathrm{Gyr}$ is plotted with a thick line. The striking result is the absence of planets in the bottom left region which corresponds to light planets (small $-E_{p}^{\prime}$ ) at short orbital distances (large $<d E_{\mathrm{EUV}} / d t>$ ). A plot of the lifetime line at $t=5 \mathrm{Gyr}$, shows that there are no planets in this part of the diagram simply because this is an evaporation-forbidden region. Planets in this region would receive more EUV energy than needed to fill the potential well of the planet, and evaporate in less than $5 \mathrm{Gyr}$, leaving a remaining core, an evaporation remnant (also named a "chthonian" planet; Lecavelier des Etangs et al. 2004).

planets, super-Earth (Santos et al. 2004) or ocean-planets (Kutchner 2003, Léger et al. 2004).

We plotted the position of these Neptune-mass planets in the energy diagram with different hypotheses on their density (Fig. 3). We used a mean planetary density of $\rho_{p}=6 \mathrm{~g} \mathrm{~cm}^{-3}$ for a typical density of refractory-rich planets which should describe the chthonian and super-Earth planets. A lower density on the order of $\rho_{p}=2 \mathrm{~g} \mathrm{~cm}^{-3}$ can be considered as more plausible for volatile-rich planets describing the ocean planets. For gas-rich planets we assumed much lower densities, $\rho_{p}=0.2 \mathrm{~g} \mathrm{~cm}^{-3}$ and $\rho_{p}=0.4 \mathrm{~g} \mathrm{~cm}^{-3}$, describing planets which should look more like irradiated Neptune-like planets. 
GJ $876 \mathrm{~d}$ cannot have the density of an ocean planet and needs to be dense enough to be located above the $t=5 \mathrm{Gyr}$ lifetime limit. This planet requires a density larger than $3.1 \mathrm{~g} \mathrm{~cm}^{-3}$ for its atmosphere to survive. GJ $876 \mathrm{~d}$ could be a big rocky planet, like a super-Earth, or a refractory remnant of a previous more massive planet (an evaporated ocean planet?).

In brief, the energy diagram allows us to trace three different categories for the presently identified Neptune-mass planets. For half of them, the EUV input energy seems not strong enough to affect significantly these planets, so that we cannot conclude on their nature. For at least three other planets (GJ 436 b, 55 Cnc e and HD 69830 b), it appears that they cannot be low-mass gaseous planets. With density necessarily above $0.5 \mathrm{~g} \mathrm{~cm}^{-3}$ to survive evaporation, these planets must contain a large fraction of solid/liquid material. Finally, GJ $876 \mathrm{~d}$ must be dense enough, with a density larger than $\sim 3 \mathrm{~g} \mathrm{~cm}^{-3}$, to survive the strong EUV energy flux from its nearby parent star. This planet must contain a large fraction of massive elements (Fig. 3).

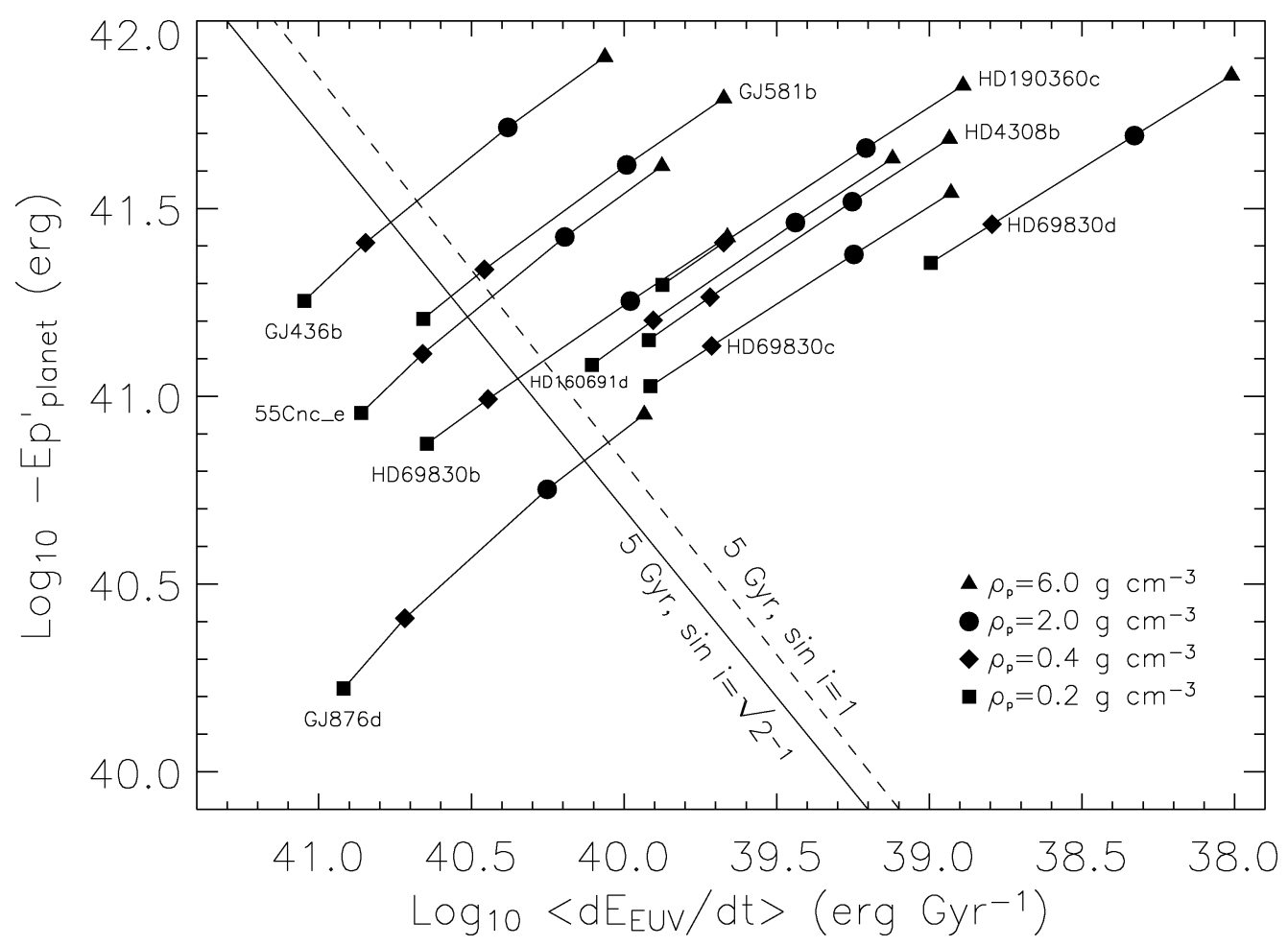

Figure 3. Plot of the potential energy of the Neptune mass planet as a function of the EUV flux for various planets' density. For GJ 581 b, GJ 436 b, HD 69830 b, 55 Cnce and GJ 876 d, and assuming $\sin i=\sqrt{2^{-1}}$, lifetime shorter than $5 \mathrm{Gyr}$ are obtained for densities below $0.28,0.55$, $0.56,0.69$ and $3.1 \mathrm{~g} \mathrm{~cm}^{-3}$, respectively. If $\sin i=1$ (dotted line), the critical (minimum) densities are increased to $0.38,0.74,0.78,0.93$ and $4.2 \mathrm{~g} \mathrm{~cm}^{-3}$, for GJ $581 \mathrm{~b}$, GJ $436 \mathrm{~b}$, HD $69830 \mathrm{~b}, 55$ Cnc e, and GJ 876 respectively. 


\section{Observations of HD $189733 \mathrm{~b}$}

The observation of the transits of HD209458b revealed that the atmosphere of this planet is hydro-dynamically escaping (Sect. 2 and 3). These observations raised the question of the evaporation state of hot Jupiters. Is the evaporation specific to HD $209458 \mathrm{~b}$ or general to hot-Jupiters? What is the evaporation mechanism, and how does the escape rate depend on the planetary system characteristics? The recent discovery of HD 189733 b, which transits a bright and nearby K0 star $(\mathrm{V}=7.7)$, offers the unprecedented opportunity to answer these questions. Indeed, among the stars harboring transiting planets, HD189733 presents the largest apparent brightness in Lyman- $\alpha$, providing capabilities to constrain the escape rate to high accuracy.

An HST program has been developed to observed HI, CII and OI stellar emission lines to search for atmospheric absorptions during the transits of HD $189733 \mathrm{~b}$. The preliminary H I Lyman- $\alpha$ transit light curve constrains the escape rate to a few $10^{8} \mathrm{~g} \mathrm{~s}^{-1}$. But a more detailed analysis of the complete data set to cover the whole light curve is needed to obtain firm conclusions.

In short, the evaporating atmosphere of only a single extrasolar planet has been detected so far. Observations of other cases under various physical conditions provide important constraints on the evaporation state and mechanisms. HD189733b being a very short-period planet orbiting a nearby late-type star with bright chromospheric emission lines, it is by far the best target to make significant progress in that field. Results are awaited for the coming months.

\section{References}

Baraffe, I., Selsis, F., Chabrier, G., et al. 2004, A\&A, 419, L13

Baraffe, I., Chabrier, G., Barman, T. S., et al. 2005, A\& $A$, 436, L47

Baraffe, I., Alibert, Y., Chabrier, G., \& Benz, W. 2006, A\& A, 450, 1221

Bouchy, F., Udry, S., Mayor, M., et al. 2005, A\&A, 444, L15

Désert, J.-M., Lecavelier des Etangs, A., Vidal-Madjar, A., Sing, D. K., et al. 2008, A\&A, submitted

Ehrenreich, D., Tinetti, G., Lecavelier Des Etangs, A., Vidal-Madjar, A., \& Selsis, F. 2006, $A \mathscr{S} A, 448,379$

Ehrenreich, D., Hébrard, G., Lecavelier des Etangs, A., et al. 2007, ApJ (Letters), 668, L179

García Muñoz, A. 2007, Planetary \& Space Science, 55, 1426

Hébrard, G. \& Lecavelier des Etangs, A. 2006, A\& $A, 445,341$

Jaritz, G. F., Endler, S., Langmayr, D., et al. 2005, A\& $A, 439,771$

Kuchner, M. J. 2003, ApJ (Letters), 596, L105

Lamers, H. J. G. L. M., Lecavelier Des Etangs, A., \& Vidal-Madjar, A. 1997, A $\& A$ A, 328, 321

Lammer, H., Selsis, F., Ribas, I., et al. 2003, ApJ (Letters), 598, L121

Lecavelier Des Etangs, A., Deleuil, M., Vidal-Madjar, A., et al. 1995, A\&A, 299, 557

Lecavelier Des Etangs, A., Vidal-Madjar, A., Burki, G., et al. 1997, A\&̊A, 328, 311

Lecavelier Des Etangs, A., Vidal-Madjar, A., \& Ferlet, R. 1999, A\& $A$, 343, 916

Lecavelier Des Etangs, A. 1999, A\&AS, 140, 15

Lecavelier des Etangs, A., Vidal-Madjar, A., Hébrard, G., \& McConnell, J. 2004, A $\&$ A, 418, L1

Lecavelier Des Etangs, A., Nitschelm, C., Olsen, E. H., Vidal-Madjar, A., \& Ferlet, R. 2005, $A \& A, 439,571$

Lecavelier Des Etangs, A. 2007, A\&A, 461, 1185

Lecavelier Des Etangs, A., Pont, F., Vidal-Madjar, A., \& Sing, D. 2008, A\&\&A, 481, L83

Lecavelier des Etangs, A., Vidal-Madjar, A., Desert, J. -., \& Sing, D. 2008b, A\&BA, 485, 865

Léger, A., Selsis, F., Sotin, C., et al. 2004, Icarus, 169, 499

Nitschelm, C., Lecavelier des Etangs, A., Vidal-Madjar, A., et al. 2000, A\&GAS, 145, 275 
Sing, D. K., Vidal-Madjar, A., Desert, J. -., Lecavelier des Etangs, A., \& Ballester, G. 2008a, ApJ, arXiv:0802.3864

Sing, D. K., Vidal-Madjar, A., Lecavelier des Etangs, A., et al. 2008b, ApJ, arXiv:0803.1054

Tian, F., Toon, O. B., Pavlov, A. A., \& De Sterck, H. 2005, ApJ, 621, 1049

Vidal-Madjar, A., Lecavelier des Etangs, A., Désert, J.-M., et al. 2003, Nature, 422, 143

Vidal-Madjar, A. \& Lecavelier des Etangs, A. 2004, ASP Conf. Ser., 321, 152

Vidal-Madjar, A., Désert, J.-M., Lecavelier des Etangs, A., et al. 2004, ApJ (Letters), 604, L69

Vidal-Madjar, A., Lecavelier des Etangs, A., Désert, J.-M., et al. 2008, ApJ, 676, 57

Yelle, R. V. 2004, Icarus, 170, 167 